\title{
Ulcerative Colitis Followed by the Development of Behçet's Disease
}

\author{
Tsuyoshi Kobashigawa, Hiroshi OKamoto, Junko Kato*, Hironari ShIndo*, \\ Tetsuo IMAMURA**, Bun-ei IIZUKA***, Masanori TANAKA****, Masashi UESATO, \\ Shu-ji OHTA, Chihiro Terai, Masako HaRA and Naoyuki Kamatani
}

\begin{abstract}
A 34-year-old man who had a history of ulcerative colitis (UC) was admitted to our hospital with complaints of arthralgia, erythema nodosum, recurrent oral aphthous ulcers and bloody stools. A colonoscopy revealed multiple aphthous ulcers on his cecum and colon and also revealed a transmural ulcer on his rectum consistent with a diagnosis of UC. The patient was HLA-B51 positive. Based on clinical evidence [recurrent oral ulcers, skin lesions (erythema nodosum), positivity for pathergy test] this patient was diagnosed as having Behçet's disease with gastrointestinal involvement. We describe this rare case of Behçet's disease with colitis and discuss the difficulties in making a differential diagnosis between Behçet's disease and the inflammatory bowel diseases.

(Internal Medicine 43: 243-247, 2004)
\end{abstract}

Key words: Behçet's disease, gastrointestinal involvement, ulcerative colitis, inflammatory bowel diseases

\section{Introduction}

Behçet's disease (BD) is a systemic inflammatory disorder of unknown etiology. BD cases are distributed worldwide, however BD is especially prevalent in Japan, the Middle East and in some Mediterranean countries $(1,2)$. Susceptibility to Behçet's disease is associated with the HLA-B51 allele in many ethnic groups (3). BD is characterized by recurrent oral aphthous ulcers, and genital ulcers, and it can be life threatening (4). Gastrointestinal Behçet's disease is a specific subtype of this disease that manifests as gastrointestinal symptoms such as nausea, vomiting, abdominal pain, diarrhea and bleeding. The lesions can be distributed along the full length of the gastrointestinal tract from the mouth to the anus. However, the ileocecal region is the most commonly affected part while the transverse colon, ascending colon, and rectum are often involved. Gastrointestinal involvement usually emerges in the course of Behçet's disease and an overlap in clinical manifestations can occur between intestinal Behçet's disease and other inflammatory bowel diseases such as Crohn's disease and ulcerative colitis (5). Here, we describe a male patient diagnosed with Behçet's disease after diagnosis with and treatment of ulcerative colitis.

For editorial comment, see p 172.

\section{Case Report}

A 34-year-old man with a history of ulcerative colitis was admitted to Aoyama Hospital, Tokyo Women's Medical University on July 12, 2000 with complaints of arthralgia in his right knee, erythema nodosum on his right leg, oral aphthous ulcers and bloody stools. Two months before his first visit, he had arthralgia in his right knee and bloody stools. After an episode of pharyngitis at the end of June 2000, his arthralgia and bloody stools worsened and he developed oral aphthous ulcers and erythema on his right leg.

At 18 years of age, the patient had bloody diarrhea and at 19 years of age, after an episode of pharyngitis, his bloody diarrhea worsened. He visited another hospital where a sigmoidscopy was performed. The sigmoidscopy showed an ulcerative lesion in his rectum. He was diagnosed with ulcerative colitis and treated with sulfasalazine $(1.5 \mathrm{~g} / \mathrm{d} \mathrm{kg})$.

From the Institute of Rheumatology, *Institute of Geriatrics and ***Institute of Gastroenterology, Tokyo Women’s Medical University, Tokyo and **the Department of Surgical Pathology, Teikyo University School of Medicine, Tokyo, **** the Second Department of Pathology, Hirosaki University School of Medicine, Hirosaki

Received for publication December 9, 2003; Accepted for publication October 9, 2003

Reprint requests should be addressed to Dr. Hiroshi Okamoto, the Institute of Rheumatology, Tokyo Women's Medical University, 10-22 Kawada-cho, Shinjuku-ku, Tokyo 162-0054 

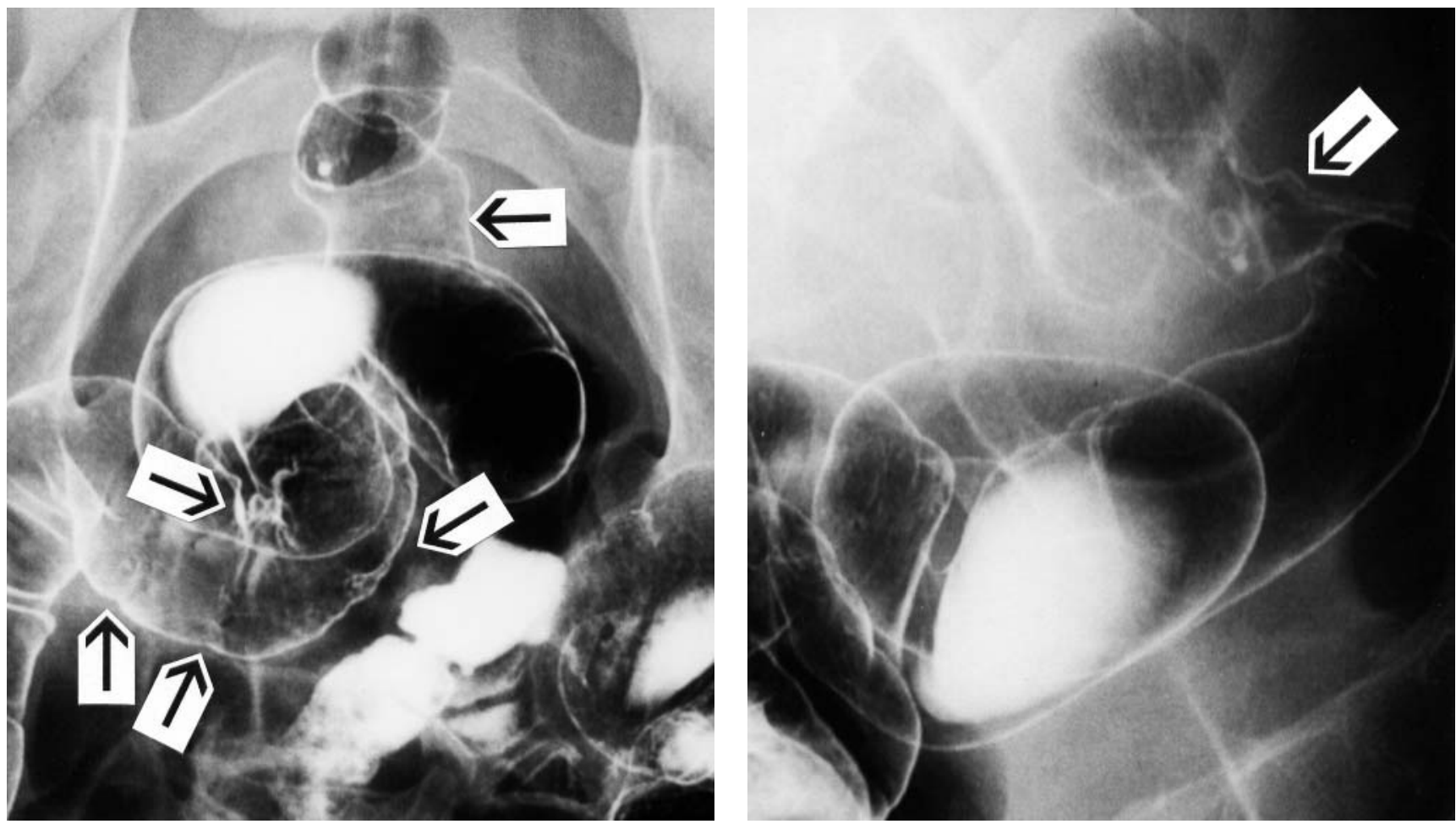

Figure 1. Results of a barium enema study. Multiple simple ulcers (arrows) in ascending colon (left panel) and the disappearance of haustral markings, resembling a "lead-pipe appearance" extended from the sigmoid colon to the rectum (right panel).

Shortly after, his symptoms disappeared.

Upon hospital admission (July 2000), the patient was 161 $\mathrm{cm}$ tall and weighed $55 \mathrm{~kg}$. Erythema nodosum was noted on his lower, right leg and arthritis was noted in his right knee joint. An aphthous ulcer was present on his upper lip but no genital ulcers were present. Neurological findings were unremarkable. An eye examination revealed no evidence of uveitis.

The patient's complete blood count showed a normal red blood cell count, mild leukocytosis $(8,400 / \mu \mathrm{l})$ and mild thrombocytosis $\left(56.9 \times 10^{4} / \mu \mathrm{l}\right)$. Blood chemistry data were within the normal range. Elevations in the erythrocyte sedimentation rate (ESR) $(48.5 \mathrm{~mm} / \mathrm{h})$ and C-reactive protein level $(2.5 \mathrm{mg} / \mathrm{dl})$ were observed. He had no detectable antinuclear antibodies. Elevation of immunoglobulin D (21.0 $\mathrm{mg} / \mathrm{dl}$ ) was observed. The pathergy test was positive. HLA typing revealed that he is HLA-B51 positive. Multiple fresh stool specimens were negative for pathogens, however, these specimens were positive for occult blood (3+). Cerebrospinal fluid revealed no abnormalities including levels of cytokines (i.e., IFN- $\gamma$, IL-6 and IL-8). Roentgenograms of his fingers, sacroiliac joints, knee joints and ankle joints showed no abnormalities.

A barium enema (Fig. 1) was obtained. It was consistent with a diagnosis of ulcerative colitis as it showed multiple simple ulcers and the disappearance of haustral markings, resembling a "lead-pipe appearance". Colonoscopy revealed multiple aphthous ulcers on the cecum, ascending colon, transverse colon, descending colon and sigmoid colon and also revealed a transmural ulcer on the rectum (Fig. 2). Biopsies of the sigmoid colon and rectum showed erosions and marked inflammatory cell infiltration in the lamina propria mucosae (Fig. 3A). The inflammatory infiltrate was composed of neutrophils, lymphocytes and plasma cells. Slight cryptitis (Fig. 3B) and moderate goblet cell depletion were apparent, but Paneth cell metaplasia was absent. Neither granulomatous lesions nor vasculitis lesions were observed. From these findings, a diagnosis of ulcerative colitis in a mildly active phase was made.

A rectal biopsy revealed moderate inflammatory cell infiltration consisting of lymphocytes and plasma cells and a moderate loss of mucosa tubules. Hyperplastic lymph follicles were prominent (Fig. 4). Neither cryptitis, crypt abscesses nor Paneth cell metaplasia were present. Thus from these findings a diagnosis of chronic, non-specific proctitis with lymphoid hyperplasia was made.

An HE staining and microscopic study revealed no cytomegalovirus inclusion bodies or amoeba. Ziehl-Nielsen staining was negative for acid-fast bacilli. The pathological characteristics of colitis in Behçet's disease are the lack of transmural inflammation, submucosal fibrosis and granulomas. On the other hand, these pathological characteristics are markers of Crohn's disease. Therefore, these pathological findings indicated the presence of entero-Behçet's disease or 


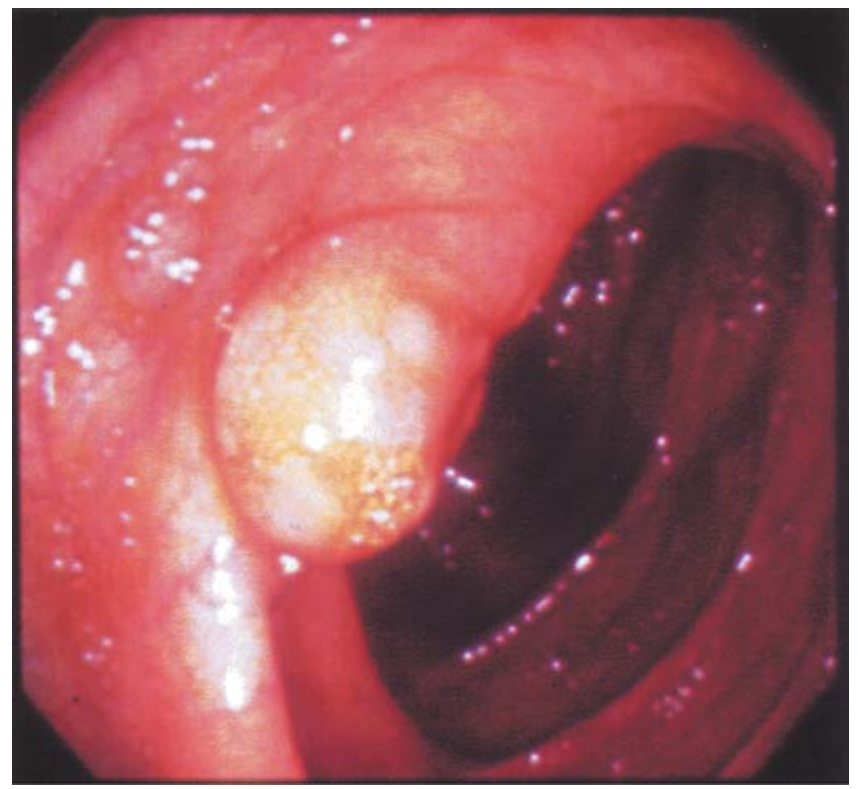

A

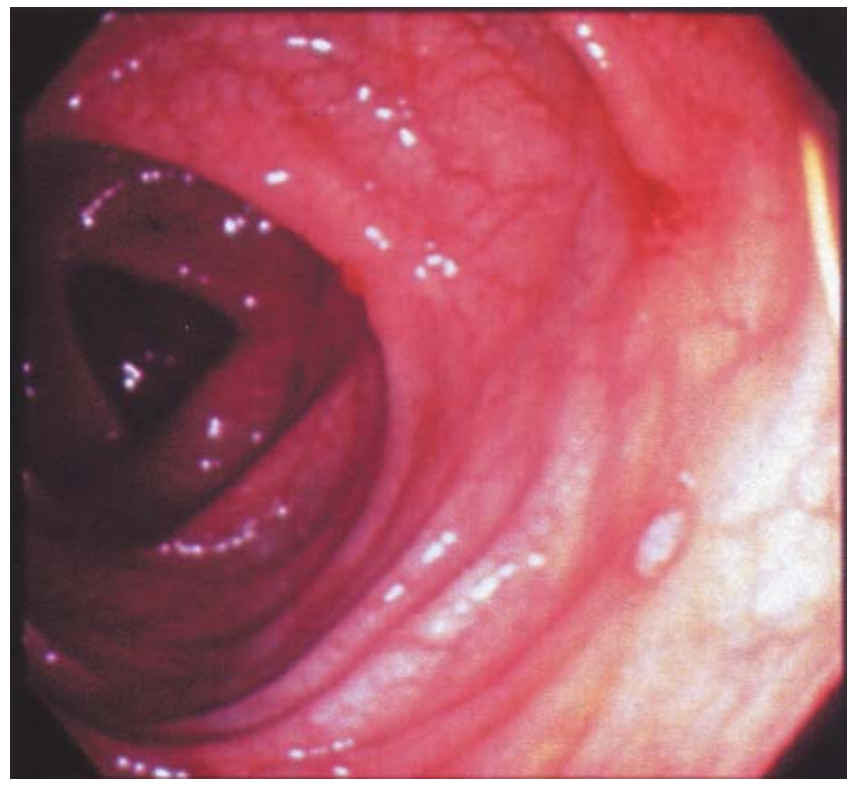

C

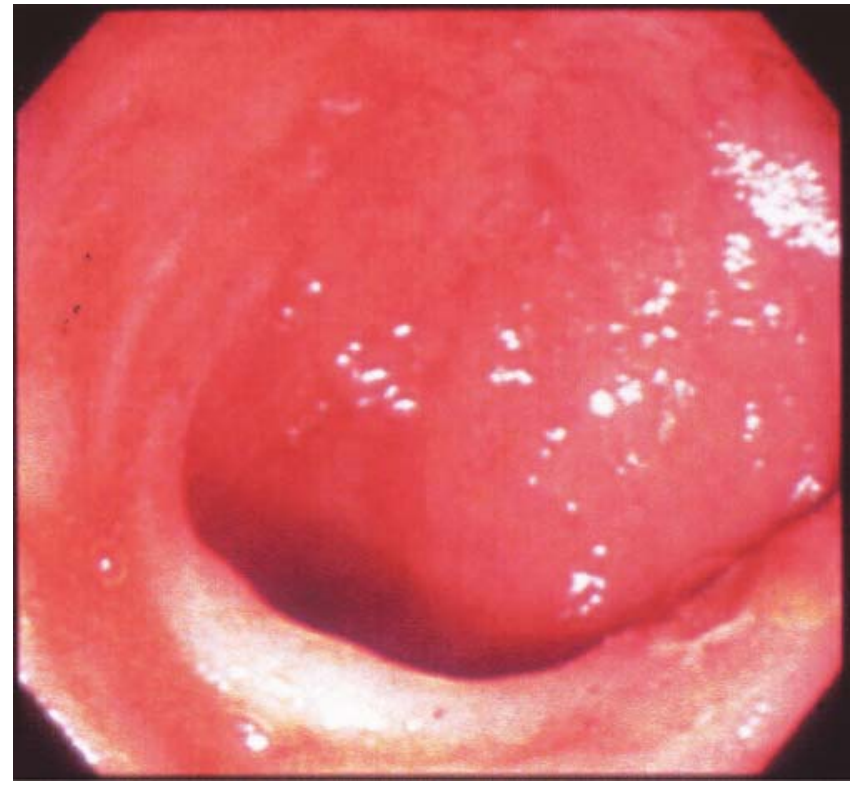

B

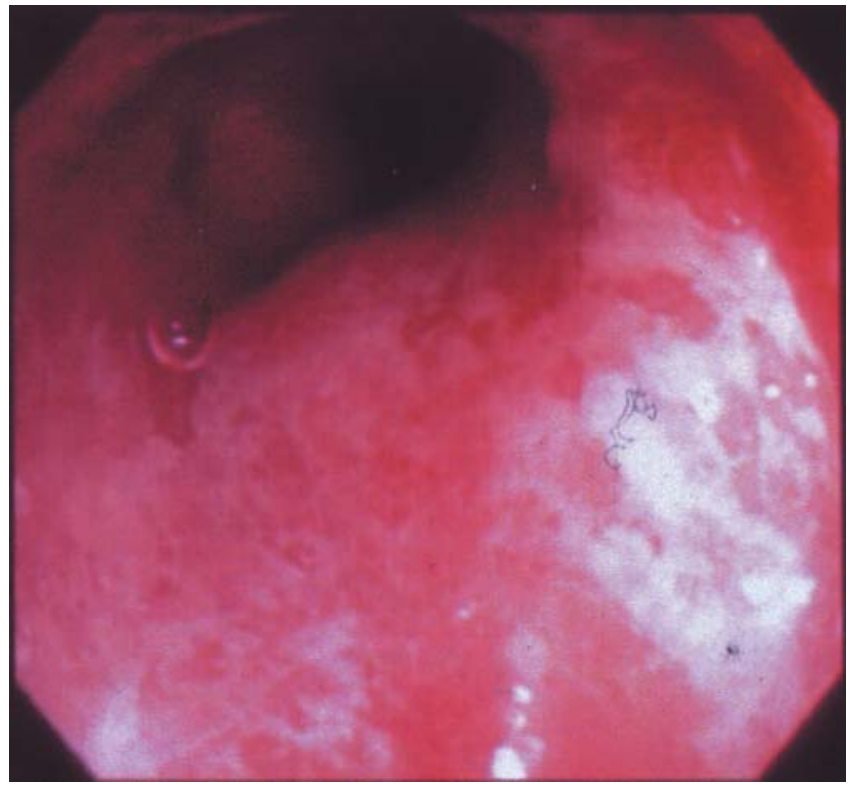

D

Figure 2. Results of a colonoscopy. Multiple aphthous ulcers on the cecum (A), ascending colon (B), transverse colon (C) and sigmoid colon (D).

Crohn's disease. Multiple fresh stool specimens failed to reveal any pathogens including $C$. difficile, Escherichia coli, Campylobacter, M. tuberculosis, Salmonella and Schistosomiasis.

Based on the following clinical evidence from this patient such as: 1) recurrent oral ulcers; 2) skin lesions (erythema nodosum); 3) positive for pathergy test; the patient was diagnosed as having Behçet's disease with gastrointestinal involvement by international criteria for diagnosis of Behcet's disease (6). However this case does not meet the standard criteria proposed by the Japan Behçet's Disease Research Committee (1987), as the case has two major symptoms (recurrent oral ulcers and skin lesions ) but only one minor symptom (arthritis without joint destruction) (7). A regimen of 5-aminosalicylate (Pentasa $1.5 \mathrm{~g} /$ day; Nisshin-Kyorin Pharma Co. Ltd., Tokyo, Japan) was started. The abdominal symptoms and genital ulcers improved after 1 month. A repeated colonoscopy revealed that the lesions including the 


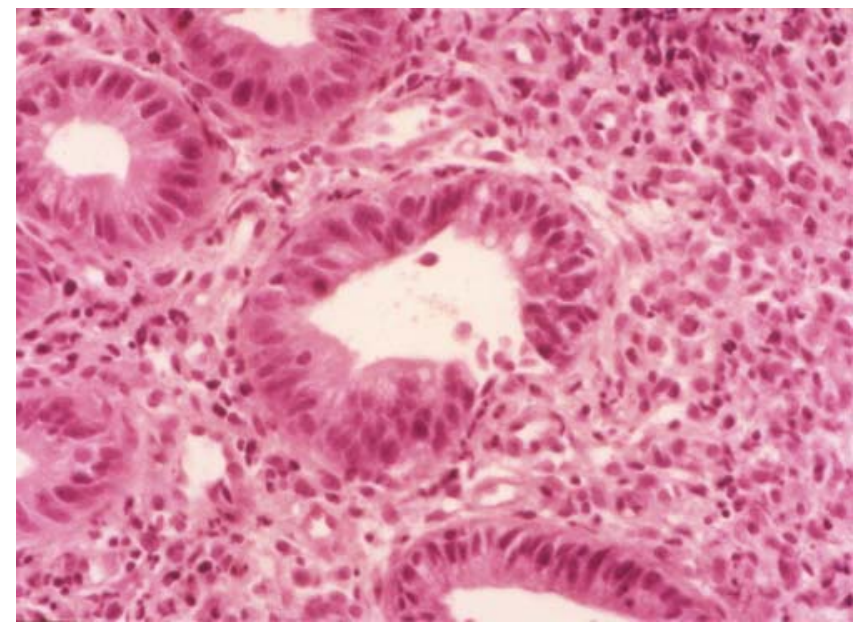

A

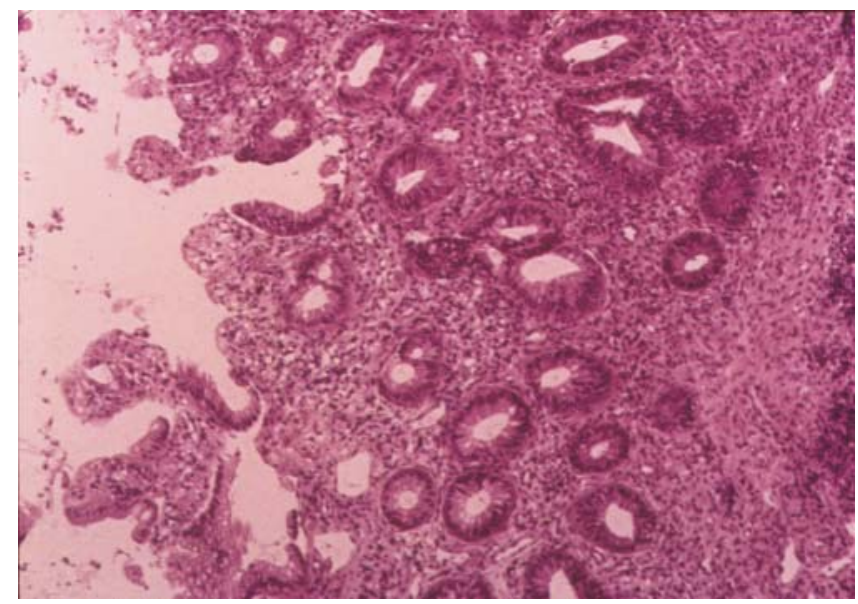

B

Figure 3. Microscopic findings of the lesion around an ulcer in the sigmoid colon. Lower panel. Erosion and marked inflammatory cell infiltration (HE stain, magnification 20x). Upper panel. Neutrophilic cell infiltration with formation of cryptitis (HE stain, magnification $66 x$ ).

multiple ulcers had also resolved. The patient was subsequently discharged 2 months after admission. 5-aminosalicylate (1.5 g/day) was continued as maintenance therapy.

\section{Discussion}

BD was initially described by Hulusi Behçet. His original description consisted of a triad of symptoms including recurrent oral and genital ulcers and relapsing iridocyclitis. Since this original description, $\mathrm{BD}$ has been shown to affect many other organs including joints, skin, blood vessels, the nervous system and the gastrointestinal tract (5, 8-10). Gastrointestinal symptoms are common in Behçet's disease and ileal ulcers, peritonitis and gastric ulcers are the most common, although colitis is rare $(11,12)$. In contrast to the ulcers

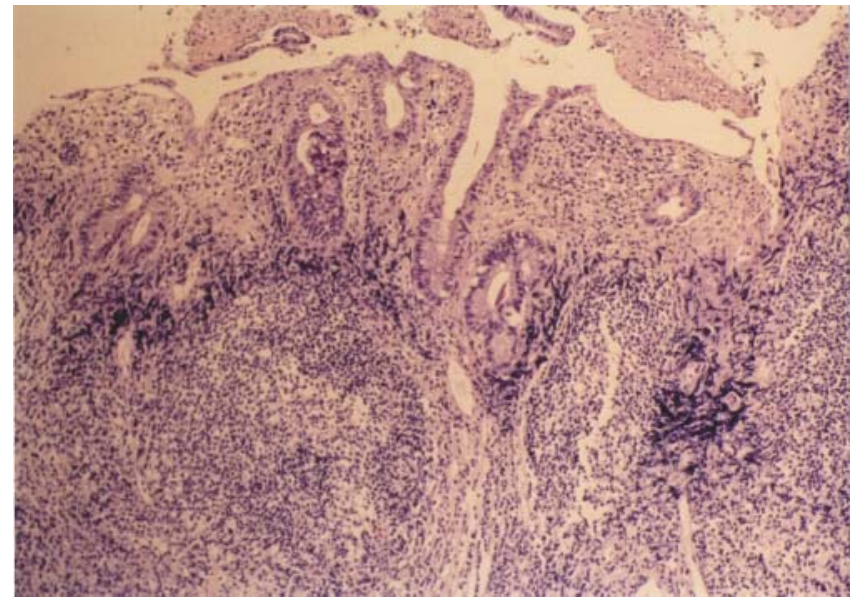

Figure 4. Biopsy from the rectum showing moderate inflammatory cell infiltration and loss of tubules as well as hyperplasia of lymph follicles (HE stain, magnification 25x).

characteristic of ulcerative colitis, the ulcers in BD are most commonly found in the terminal ileum and cecum (75\%) and less frequently in the colon, sparing the rectum (3\%) (13). Intestinal lesions in Crohn's disease are characterized by the presence of longitudinal ulcers with a cobblestone appearance and non-caseating epithelioid granulomas. Colitis in Behçet's disease is characterized by round and oval punched out ulcers that are seldom accompanied by granulomas. Thus, the case presented here can be distinguished from Crohn's disease.

Ulcerative colitis is one of the inflammatory bowel diseases. Arthritis, uveitis, oral aphthous ulcers, erythema nodosum and venous thromboembolism are typical clinical features of Behçet's disease. However, these symptoms also occur in $1 \%$ to $39 \%$ of patients with inflammatory bowel diseases $(14,15)$. Thus far, there are no reports showing the presence of lead-pipe appearance in colitis in Behçet's disease. And also there are no reports presenting patients diagnosed with Behçet's disease more than 15 years after the diagnosis of ulcerative colitis. Therefore, it is difficult to determine whether the patient did in fact have Behçet's disease with ulcerative colitis-like lesions, or ulcerative colitis with BD-like extraintestinal complications (i.e., arthritis, oral aphthous ulcers and erythema nodosum), or both Behçet's disease and ulcerative colitis. From these similarities between Behçet's disease and ulcerative colitis, mutual factors such as infectious agents must be involved in the pathogenesis of both diseases. The present patient did, however, have some specific features of Behçet's disease including positive for pathergy test, expression of HLA-B51. As HLA-B51 has never been reported to be associated with ulcerative colitis, rather significant association of B52 and DR2 with UC was reported $(16,17)$. Accordingly, we gave a diagnosis of Behçet's disease concomitant with ulcerative colitis.

In summary we have described a patient with Behçet's 


\section{Behçet's with UC-like Enteropathy}

disease accompanied by colonic lesions (i.e., multiple simple ulcers and the disappearance of haustral markings, resembling a "lead-pipe appearance" by barium enema and multiple aphthous ulcers on the cecum, ascending colon, transverse colon, descending colon and sigmoid colon as well as a transmural ulcer on the rectum revealed by colonoscopy) which was difficult to distinguish from ulcerative colitis. After one year from the initial hospitalization, his ulcerative lesions recurred on the sigmoid colon and he was given corticosteroid ( $40 \mathrm{mg} /$ day) and thereafter the ulcerative lesions disappeared. The patient has been followed for 2 years and to date, corticosteroid was gradually tapered to 4 $\mathrm{mg} /$ day and 5 -aminosalicylate $(1.5 \mathrm{~g} /$ day $)$ was continued as maintenance therapy, and the intestinal lesions have improved with slight recurrence of oral aphthous ulcers and no indications of ulcerative colitis or erythema have become apparent.

\section{References}

1) Kastner DL. Intermittent and periodic arthritic syndromes. in: Arthritis and Allied Conditions: a Textbook of Rheumatology. 13th ed. Vol. 1. Koopman WJ, Ed. Williams \& Wilkins, Baltimore, 1997: 1279-1306.

2) Sakane T, Takeno M, Suzuki N, Inaba G. Behçet's disease. N Engl J Med 341: 1284-1291, 1999.

3) Mizuki N, Inoko H, Ohno S. Pathogenic gene responsible for the predisposition to Behçet's disease. Int Rev Immunol 14: 33-48, 1997.

4) Dilsen N, Konice M, Aral O, et al. Risk factors for vital organ involvement in Behçet's disease. in: Behçet's Disease. Wechsler B, Godeau P, Ed. Excerpta Media, Amsterdam, 1993: 165-169.
5) Yim CW, White RH. Behçet's syndrome in a family with inflammatory bowel disease. Arch Intern Med 145: 1047-1050, 1985.

6) International Study Group for Behçet's Disease. Criteria for diagnosis of Behçet's disease. Lancet 335: 1078-1080, 1990.

7) Mizushima Y. Revised diagnostic criteria for Behçet's disease in 1987. Ryumachi 28: 66-70, 1988.

8) Bang D. Clinical spectrum of Behçet's disease. J Dermatol 28: 610613, 2001.

9) Kasahara Y, Tanaka S, Nishino M, Umemura H, Shiraha S, Kuyama T. Intestinal involvement in Behçet's disease: review of 136 surgical cases in the Japanese literature. Dis Colon Rectum 24: 103-106, 1981.

10) Yazici H, Yurdakul S, Hamuryudan V. Behçet disease. Curr Opin Rheumatol 13: 18-22, 2001.

11) Smith GE, Kime LR, Pitcher JL. The colitis of Behçet's disease: a separate entity? Colonoscopic findings and literature review. Am J Dig Dis 18: 987-1000, 1973

12) Lakhanpal S, Tani $K$, Lie JT, Katoh $K$, Ishigatsubo $Y$, Ohokubo T. Pathologic features of Behçet's syndrome: a review of Japanese autopsy registry data. Hum Pathol 16: 790-795, 1985.

13) Sayek I, Aran O, Uzunalimoglu B, Hersek E. Intestinal Behçet's disease: surgical experience in seven cases. Hepatogastroenterology 38 : 81-83, 1991.

14) Shimizu T, Ehrlich GE, Inaba G, Hayashi K. Behçet's disease (Behçet's syndrome). Semin Arthritis Rheum 8: 223-260, 1979.

15) Kern F Jr. Extraintestinal complications. in: Inflammatory Bowel Disease. Kirsner JB, Shorter RG, Eds. Lea \& Febiger, Philadelphia, 1975: 127-145.

16) Seki SS, Sugimura K, Ota M, et al. Stratification analysis of MICA triplet repeat polymorphisms and HLA antigens associated with ulcerative colitis in Japanese. Tissue Antigens 58: 71-76, 2001.

17) Binder V, Orholm M. Familial occurrence and inheritance studies in inflammatory bowel disease. Neth J Med 48: 53-56, 1996. 